\title{
Gene Expression Regulation by Vitamin D Receptor Pathway
}

National Cancer Institute

\section{Source}

National Cancer Institute. Gene Expression Regulation by Vitamin D Receptor Pathway.

NCI Thesaurus. Code C39263.

A pathway that modulates the expression of genes involved in calcium homeostasis, bone formation, cellular differentiation, cell growth and apoptosis through the direct interaction between the nuclear receptor, vitamin D3 receptor (VDR), primarily as part of a heterodimeric complex with the retinoid X receptor (RXR), and vitamin $D$ response elements (VDREs) found in the promoter regions of vitamin D regulated genes. 